\title{
Deposit buildup on prosthetic eyes and implications for conjunctival inflammation and mucoid discharge
}

This article was published in the following Dove Press journal:

Clinical Ophthalmology

30 October 2012

Number of times this article has been viewed

\author{
Keith Raymond Pine' \\ Brian Sloan ${ }^{2}$ \\ Robert John Jacobs' \\ 'Department of Optometry and Vision \\ Science, ${ }^{2} \mathrm{New}$ Zealand National Eye \\ Centre, The University of Auckland, \\ Auckland, New Zealand
}

Background: The aim of this study was to investigate deposit buildup on prosthetic eyes and the implications for conjunctival inflammation and discharge.

Methods: Forty-three prosthetic eye wearers participated in the study. Twenty-three had their prostheses polished normally before being worn continuously for 2 weeks. After this time, surface deposits were stained, photographed, and graded. The prostheses were then repolished to optical quality contact lens standard and worn for a further 2 weeks, when the deposits were again stained, photographed, and graded. Two participants had deposits on their prostheses stained, photographed, and graded on nine occasions at decreasing intervals ranging from 1 year to 1 day. Eighteen participants had the wetting angles on their prostheses measured with a goniometer before and after cleaning, after polishing normally, after polishing to optical quality contact lens standard, and after 10 minutes of wearing their optical quality contact lens polished prostheses. Concordance correlation, multiple regression, and paired $t$-tests were used for the statistical analysis.

Results: More surface deposits accumulated on prostheses polished normally than on those polished to an optical quality contact lens standard after 2 weeks of wear. The interpalpebral zone of most prostheses (observed without magnification) appeared to be clear of deposits. Removal of deposits significantly decreased surface wettability, but wettability returned after 10 minutes of wear. Optical quality contact lens polishing produced more wettable surfaces and a slower rate of deposit accumulation than normal polishing.

Conclusion: We recommend that an optical quality contact lens standard be the minimum standard of finish for prosthetic eyes. This standard may assist the smooth action of the lids over the interpalpebral zone of the prosthesis and the cleansing action of tears. The presence of deposits in the retropalpebral zone may improve the lubricating properties of socket fluids which, in turn, may result in less frictional irritation of the conjunctiva and less mucoid discharge.

Keywords: prosthetic eye, deposits, wettability, conjunctival inflammation, mucoid discharge, cleaning regime

\section{Introduction}

Pine et al have used recently developed measuring tools ${ }^{1}$ to demonstrate that the presence of surface deposits on prosthetic eyes is associated with less conjunctival inflammation and less severe mucoid discharge in anophthalmic sockets. ${ }^{2}$ However, the causal direction of these associations was not established in this study. Furthermore, there appears to be no literature describing deposit buildup on prosthetic eyes, even though extensive literature describes deposit buildup on contact lenses. Prosthetic eyes are somewhat analogous to contact lenses, but are made from different materials and worn for very different reasons. Both devices come into contact with the conjunctiva,
Correspondence: Keith Raymond Pine New Zealand National Eye Centre, Department of Optometry and Vision Science, The University of Auckland, Private Bag 92019, Auckland II42,

New Zealand

Tel +6493737599

Email keith.pine@kp-assoc.co.nz 
share a similar eyelid action, are bathed in the same ocular fluids, and accumulate surface deposits. Because of these similarities, relevant information from contact lens investigations provides a useful background for this study. For example, the composition of deposits on contact lenses is likely to be similar to deposits on prosthetic eyes. Contact lens deposits include tear proteins, lipids (lipid deposit buildup may be both on the surface and inside the lens matrix ${ }^{3,4}$ ), mucin, and contaminants, such as skin lipids, dirt, microorganisms, and metallic and nonmetallic debris. ${ }^{5}$

The aims of this investigation were: to describe the formation of surface deposits on prosthetic eyes over time; to investigate rates of deposit buildup on prostheses with different standards of polish; and to understand the cause of the reported associations between deposits, conjunctival inflammation, and severity of mucoid discharge. ${ }^{1}$

\section{Materials and methods}

Forty-three unilateral prosthetic eye wearers were entered into the study after they completed a questionnaire and agreed to participate in prosthetic eye research which had prior approval from the University of Auckland Human Participants Ethics Committee and the Multi-Region Ethics Committee of the New Zealand Ministry of Health. Participants were excluded from the study if they were aged younger than 18 years, had ocular health issues, or had not worn a prosthetic eye for at least 6 months.

\section{Measuring surface deposits on prosthetic eyes}

A staining solution was made by dispersing $5 \mathrm{~g}$ of plaque disclosing gel (a mix of mainly ethyl alcohol, food red 105, and water; GC Corporation, Tokyo, Japan) in $30 \mathrm{~mL}$ of $0.85 \%$ saline solution. ${ }^{1}$ The participants' prostheses were submerged in the solution at $20^{\circ} \mathrm{C}\left(68^{\circ} \mathrm{F}\right)$ for a period of 2 minutes. After removing and blotting with tissue paper, the prosthetic eyes were photographed front and back against a black background which included a standard gray scale and a color scale to ensure the consistency of photographic settings throughout the study. Standardized camera settings were used. ${ }^{2}$ Photographs of the stained prostheses were coded to deidentify the participant but to allow tracking. Each photograph was copied onto the center of a Microsoft PowerPoint (Microsoft Corporation, Redmond, WA) slide which contained a previously developed 0-10 photographic grading scale by which the extent and intensity of deposit buildup could be measured. ${ }^{1}$ Separate anterior surface and posterior surface deposit scales allowed assessment of the severity of deposit buildup according to the extent and intensity of the stained deposits. The graders were the authors (an experienced ophthalmologist, optometrist, and ocular prosthetist) who used interpolated grades to the nearest 0.1 between 0 and 10 when assessing the deposits. The final grade for the stained deposits on each prosthetic eye was the average of the three graders' anterior surface and posterior surface scores.

\section{Polishing prosthetic eyes}

The process for polishing prosthetic eyes in this study involved four steps. Firstly, the prosthesis was trimmed and the surfaces ground all over with a fine (120 grit) arbor band. Secondly, diatomaceous earth was applied to the surfaces with a wet calico polishing mop and/or a felt cone to remove the marks left by the arbor band. Thirdly, polishing compound for plastics final polish (Bego, Lincoln, $\mathrm{RI}^{6}$ ) was applied with a dry calico polishing mop to achieve the normal polish grade. The Bego product is no longer in production but is similar to other commercially available denture polishing compounds. Fourthly, an optical quality contact lens polishing standard was obtained using aluminum oxide paste applied with a foam polyurethane rotating cone.

\section{Deposit buildup on prosthetic eyes polished to normal and to optical quality contact lens standards}

Twenty-three of the 43 participants enrolled in the study had their prostheses finished to a normal polishing standard before return to the socket where they were worn without removing and cleaning for 2 weeks. At the end of the 2-week period, the prostheses were removed and their surface deposits were graded according to the method for measuring surface deposits on prosthetic eyes described above. The prostheses were then cleaned and polished to an optical quality contact lens standard before being returned to the participants and worn continuously for a further 2 weeks. At this point, they were removed and their surface deposits were graded as before. Concordance correlation $^{7}$ and paired $t$-tests were used to investigate differences after 2 weeks of continuous wear between the grade of deposit buildup on prosthetic eyes polished to normal standard and to optical quality contact lens standard.

\section{Deposit buildup on prosthetic eyes worn continuously over time}

Two of the 43 participants were chosen because their prostheses had not been removed and cleaned for 12 months. Their prostheses were removed and the surface deposits were measured according to the method for measuring 
surface deposits on prosthetic eyes described above. The stained deposits were removed by wiping them firmly with a paper towel wetted with cold water before being returned to the participants for continued wear without removing or cleaning. This measuring process was repeated eight more times at decreasing intervals ranging from one month to one day. The extent and intensity of the stained deposits at each interval was plotted as a function of time, and regression analysis was used to describe the results further.

\section{Wettability of prosthetic eyes under different conditions of wear}

The final 18 of the 43 participants were requested to not remove or clean their prostheses for at least 24 hours. After this time, their prostheses were removed and gently blotted dry with tissue paper before being placed in a goniometer. Distilled water droplets were applied to the least convex area that could be found on each prosthesis (usually just on or above the superior limbus). This slightly convex area was adjusted to be as horizontal as possible. Wetting angles were assessed and the average of right and left angles made by each droplet of water was calculated for each prosthetic eye. High wetting angles indicated low wettability and vice versa.

The prostheses were wiped clean of deposits with a wetted paper towel and the wetting angles were measured again. They were measured a third time after the prostheses were polished to a normal standard (as described earlier in the methods used for polishing prosthetic eyes), and a fourth time after the prostheses were polished to an optical quality contact lens standard. The prosthetic eyes were then returned to the participants and ten of them wore their prostheses for 10 minutes before returning to have the wetting angles on their prosthetic eyes measured a fifth time. Multiple regression was the statistical method used to analyze the data.

\section{Results}

\section{Deposit buildup on normal and highly polished prosthetic eyes}

The rate of deposit buildup was greater on normally polished prostheses than on prostheses polished to optical quality contact lens standard. After 2 weeks of continuous wear, the normally polished prostheses had significantly more deposits (mean $3.06 \pm 1.91$ ) than prostheses polished to optical quality contact lens standard (mean $2.26 \pm 2.00$, paired-samples $t$-test, mean difference $-0.81 \pm 1.40,95 \%$ confidence intervals -1.48 to $-0.13, P=0.02$ ). Two qualitative examples are shown in Figure 1.

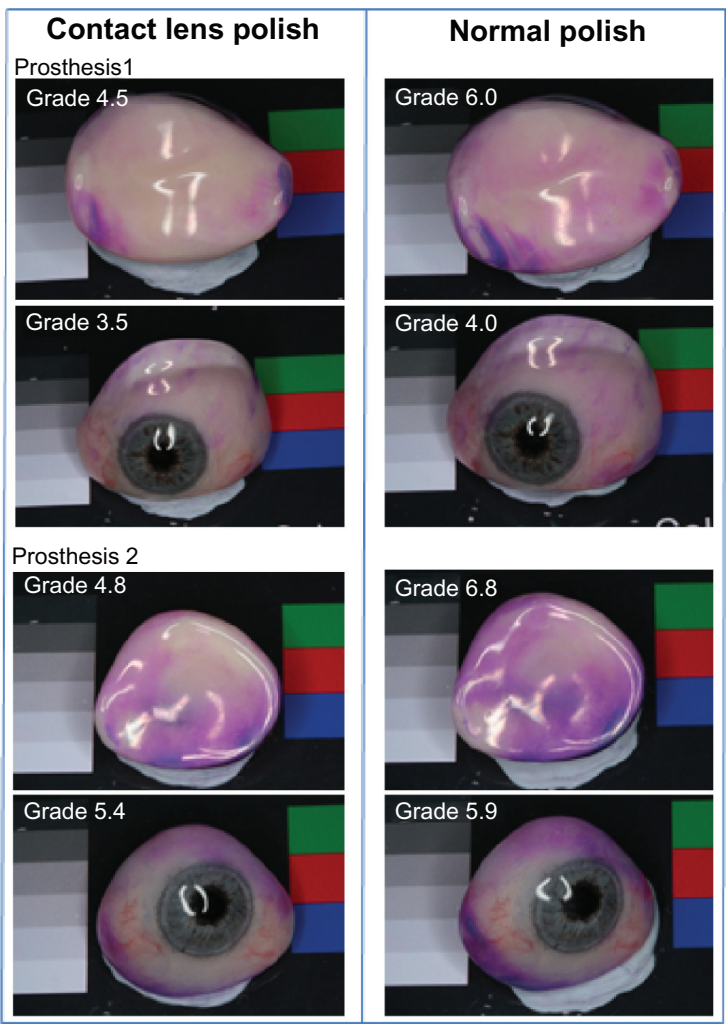

Figure I Deposit formation on the anterior and posterior surfaces of two prosthetic eyes.

Notes: The prostheses were first cleaned and polished normally and worn for 2 weeks. They were then cleaned and polished to optical quality contact lens standard and worn for a further 2 weeks. Prosthesis I was worn by a 75-year-old man. Prosthesis 2 was worn by a 77-year-old woman. The grades were measured using equal interval photographic grading scales. ${ }^{2}$

\section{Deposit buildup on prosthetic eyes worn continuously over time}

Figure 2 shows deposit buildup over time. Regression analysis results (coefficient of determination $\mathrm{R}^{2}=0.98$, residual standard deviation $=0.4, P<0.0001$ ) are shown in Figure 3. The photographic series in Figure 4 illustrates the rate of buildup of deposits on the surface of the prosthetic eye worn by participant 2 in Figure 2. A notable characteristic of deposit formation indicated by the photographs is that the interpalpebral zone (observed without magnification) remained clear of stained deposits for considerable periods of continuous wear. However, it was observed that deposits could encroach onto this space after prolonged periods. An example of this is shown in Figure 5.

\section{Wettability of prosthetic eyes under different conditions of wear}

The wetting angles of prosthetic eyes when first removed from the socket increased significantly when the eyes were wiped clean with a paper towel wetted with cold water 


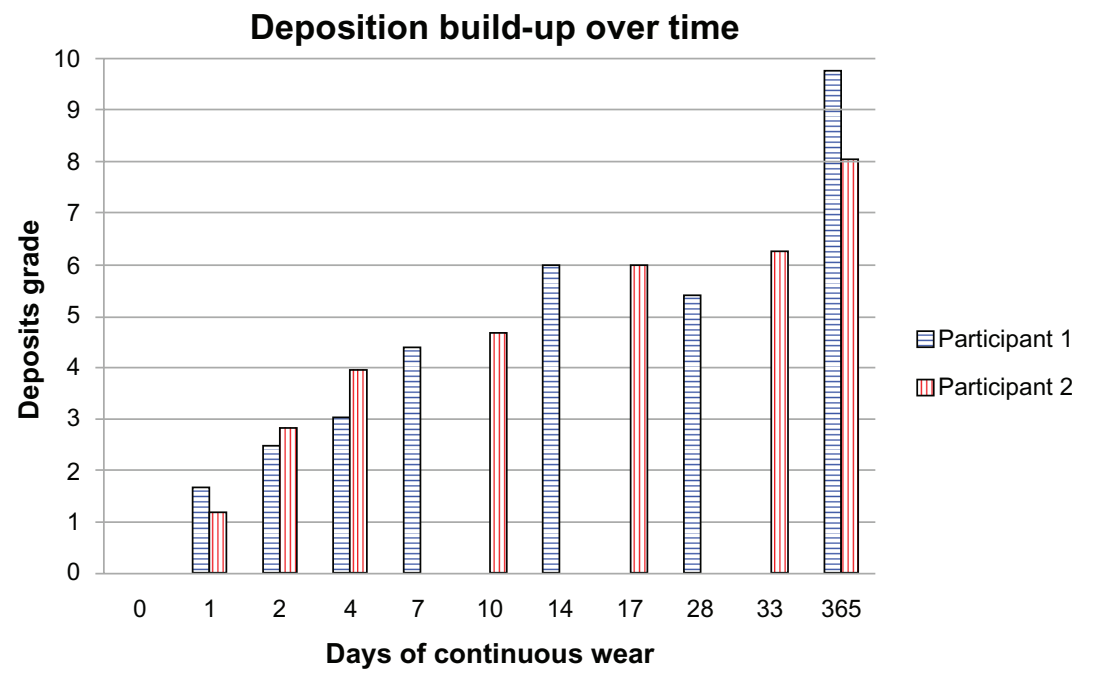

Figure 2 Deposit grades on prosthetic eyes worn continuously by two participants over the times indicated. Notes: See Figure 3 for regression analysis. The prosthetic eye worn by participant two is shown in Figure 4.

$(P<0.0001)$ and when they were polished to a normal standard $(P<0.0001)$. The increased wetting angles indicated that the wettability of prosthetic eye surfaces was reduced when deposits were removed and was reduced further still when the prostheses were polished to a normal standard.

However, the wetting angles of prosthetic eyes when first removed from the socket were not significantly different to the wetting angles of prostheses freshly polished to an optical quality contact lens standard $(P=0.117)$ or to wetting angles 10 minutes after the prosthetic eyes were placed back in their sockets $(P=1.0$; Table 1 and Figure 6). These wetting angle measurements indicate that the wettability of the prosthetic eye surfaces was the same for prostheses worn continuously for at least 24 hours, for prostheses polished to an optical quality contact lens

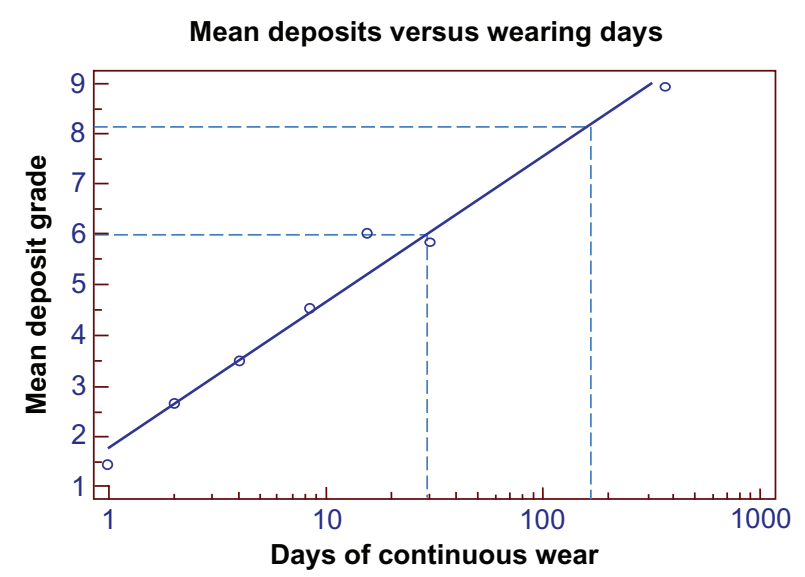

Figure 3 Regression analysis for mean extent and intensity of stained deposits on prosthetic eyes worn continuously by two participants over time.

Notes: Regression line shows mean deposit grade $=1.8+2.9 \log$ (days). Estimated mean deposit grades at one month and 6 months are depicted by dashed lines. standard before wear, and for freshly polished prostheses after 10 minutes in the socket.

\section{Discussion}

Before the introduction of hydrogel (soft) and rigid gas permeable lenses, almost all contact lenses were made from poly-methyl methacrylate (PMMA), which is the same material used for manufacturing prosthetic eyes. Deposits on PMMA contact lenses are similar in form to deposits on soft lenses, ${ }^{8}$ but no comparisons between deposits on PMMA lenses and PMMA prosthetic eyes are available. Fowler et $\mathrm{al}^{8}$ used scanning electron microscopy to investigate deposits on the anterior surfaces of PMMA contact lenses. They found that deposits on PMMA lenses were readily removed with a single cleaning and that PMMA lenses attracted fewer deposits
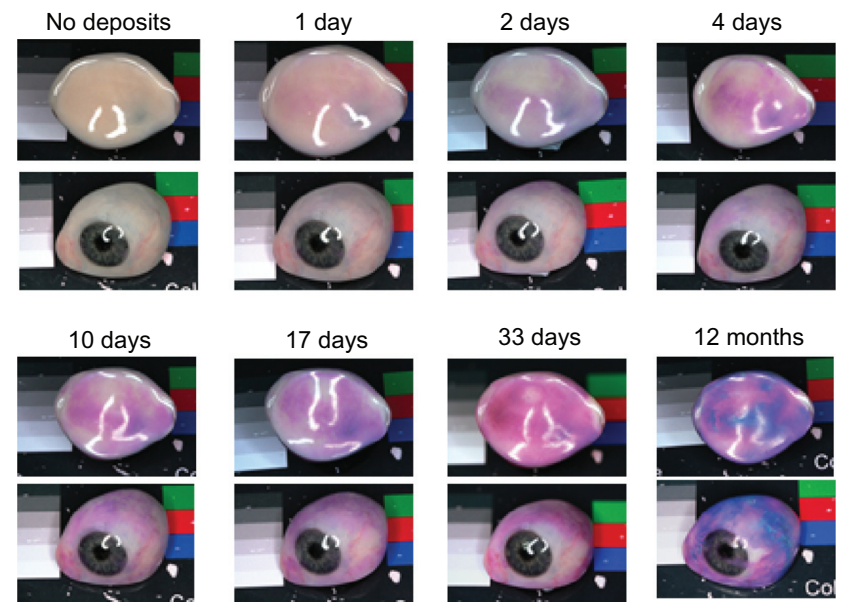

Figure 4 Photographic series showing formation of deposits on the anterior and posterior surfaces of a normally polished prosthetic eye worn continuously for the number of days indicated. 


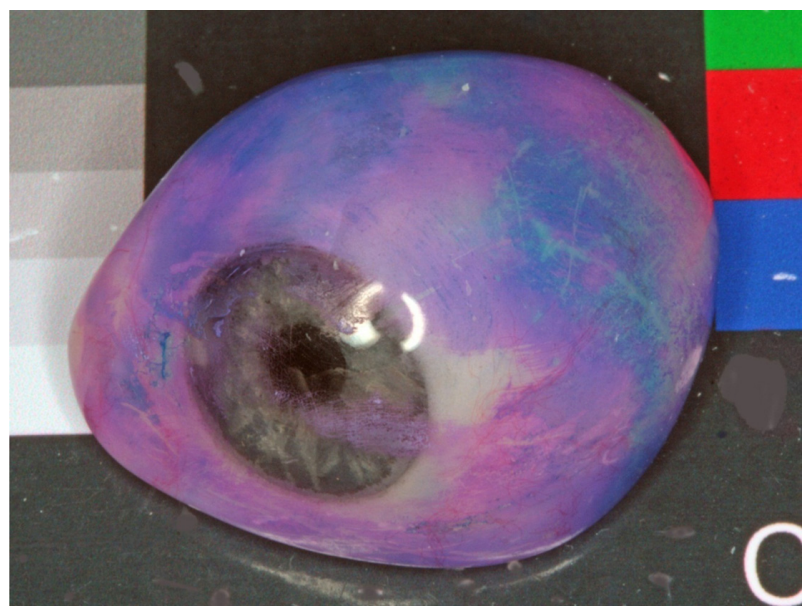

Figure 5 Deposits encroaching on the interpalpebral zone after I year of continuous wear.

than soft lenses. The layered coatings, films, or plaques of tear protein deposits described by Franklin et $\mathrm{al}^{9}$ appear to be similar to the most prevalent type of deposits on prosthetic eyes. They mainly occupy the surfaces that are in continuous contact with the conjunctiva and can be seen in Figure 7, which depicts dry stained deposits on the temporal limbus of a left prosthetic eye worn continuously for 3 months (magnification $400 \times)$.

Two distinct zones of surface deposit buildup on prosthetic eyes were observed in this study. The first was the interpalpebral zone, where deposits are exposed to air and the action of the eyelids, and the second was the retropalpebral zone, where deposits are in continuous contact with the conjunctiva. These areas are clearly shown in Figure 1, prosthesis 2 . While the interpalpebral zone appeared to be clear of deposits, microscopic analysis was not used in this study. If deposits are present in the interpalpebral zone, they are likely to be the same or similar to the deposits on contact lenses described by Fowler et $\mathrm{al}^{8}$ and Franklin et al. ${ }^{9}$ Issues such as inefficient or incomplete blinking are likely to influence deposit buildup in the interpalpebral zone, but not on other areas of the prosthesis.

The normal polishing technique described in this study is the usual standard of finish for dentures and most prosthetic eyes (at least in New Zealand), while the optical quality contact lens polish is the technique commonly used for polishing hard contact lenses. Optical quality contact lens polish has been recommended for prosthetic eye polishing by LeGrand. ${ }^{10}$ The finding that prosthetic eyes polished to an optical quality contact lens standard accumulated deposits at slower rates than normally polished prosthetic eyes suggests that adherence of deposits depends on the relationship between surface matrix fineness and the size of protein molecules, and that the surface matrix of normally polished prosthetic eye surfaces enables protein molecules to adhere more readily than finer polishes. Surface matrix fineness also appears to be a factor influencing deposition on contact lenses. For example, Franklin et al reported that one of the reasons that deposits take longer to build up on rigid gas permeable lenses than on hydrogel lenses is that rigid gas permeable polymers have lower matrix porosity. ${ }^{3}$

Table I Mean wetting angles of 18 prosthetic eyes after different interventions

\begin{tabular}{|c|c|c|c|c|c|c|}
\hline \multirow[t]{2}{*}{ Surface } & \multirow[t]{2}{*}{ Intervention } & \multirow[t]{2}{*}{ Mean difference ${ }^{* *}$} & \multirow[t]{2}{*}{ Standard error } & \multirow[t]{2}{*}{$P$-value } & \multicolumn{2}{|c|}{$95 \%$ confidence interval } \\
\hline & & & & & Lower bound & Upper bound \\
\hline \multicolumn{7}{|l|}{ Multiple regression } \\
\hline \multirow[t]{4}{*}{ Original condition } & After cleaning & $-62.05^{*}$ & 8.07 & $<0.0001$ & -85.08 & -39.02 \\
\hline & Normal polish & $-41.97^{*}$ & 8.07 & $<0.0001$ & -64.99 & -18.94 \\
\hline & Contact lens polish & -20.98 & 8.22 & 0.117 & -44.42 & 2.45 \\
\hline & After 10 minutes of wear & 2.6 & 8.14 & 1.000 & -20.64 & 25.81 \\
\hline \multirow[t]{4}{*}{ After cleaning } & Original condition & $62.05^{*}$ & 8.07 & $<0.0001$ & 39.02 & 85.08 \\
\hline & Normal polish & 20.08 & 8.00 & 0.132 & -2.75 & 42.9 \\
\hline & Contact lens polish & $41.07^{*}$ & 8.15 & $<0.0001$ & 17.82 & 64.31 \\
\hline & After 10 minutes of wear & $64.63^{*}$ & 8.07 & $<0.0001$ & 41.6 & 87.66 \\
\hline \multirow[t]{4}{*}{ Normal polish } & Original condition & $41.97^{*}$ & 8.07 & $<0.0001$ & 198.94 & 65 \\
\hline & After cleaning & -20.07 & 8.00 & 0.132 & -42.9 & 2.75 \\
\hline & Contact lens polish & 21.99 & 8.16 & 0.110 & -2.26 & 44.23 \\
\hline & After 10 minutes of wear & $44.55^{*}$ & 8.07 & $<0.0001$ & 21.52 & 67.58 \\
\hline \multirow[t]{4}{*}{ Contact lens polish } & Original condition & 20.98 & 8.22 & 0.117 & -2.46 & 44.43 \\
\hline & After cleaning & $-41.07^{*}$ & 8.15 & $<0.0001$ & -64.31 & -17.82 \\
\hline & Normal polish & -20.98 & 8.15 & 0.110 & -44.23 & 2.26 \\
\hline & After 10 minutes of wear & $23.56^{*}$ & 8.22 & 0.048 & 0.12 & 47.01 \\
\hline
\end{tabular}

Notes: High wetting angles indicate low wettability and vice versa. A minus sign $(-)$ denotes negative values. *Statistically significant; ** difference between conjunctival inflammation in the anophthalmic socket and the companion eye. 
Wetting angles on artificial eyes after interventions

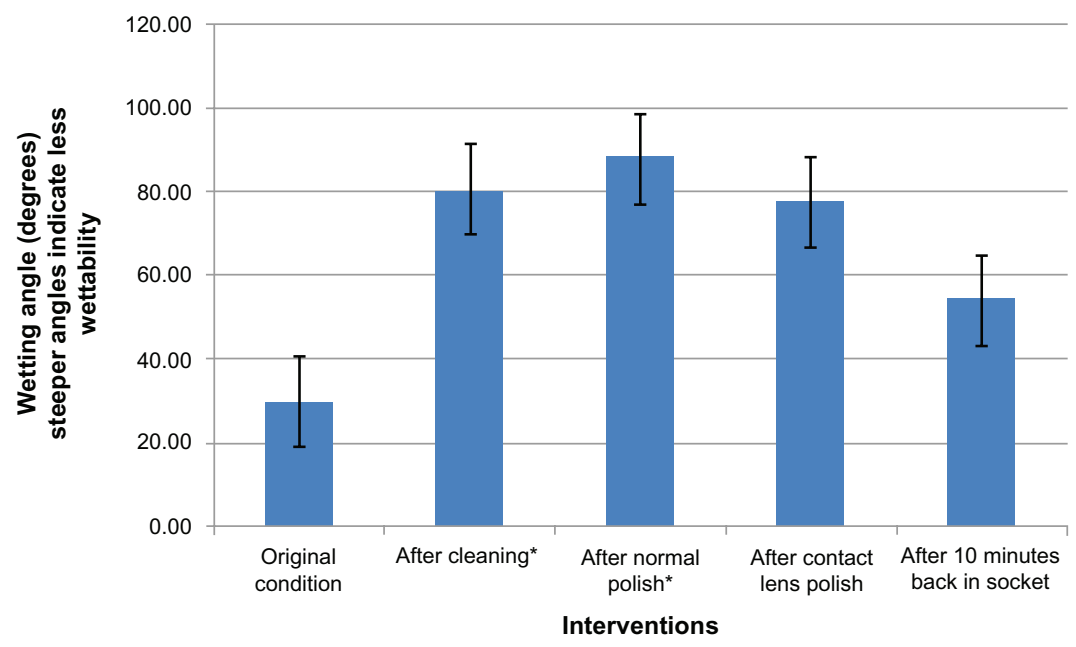

Figure 6 Wetting angles measured with a goniometer after different interventions.

Notes: Measurements made using the slightly convex "scleral" area near the superior limbus of the prostheses. Standard error bars are included. See Table I for statistical data. *Indicates significant difference from original condition.

Surface wettability is another factor that influences deposition on contact lenses, with increasing wettability having been shown to decrease deposition. ${ }^{11}$ A goniometer was used in this study for measuring wetting angles on prosthetic eyes. Wetting angle (or contact angle) analysis has become a widely accepted method by which to infer the wetting characteristics of contact lenses. It involves measuring the angle between a liquid and the lens surface at the three-phase boundary where a liquid, gas, and solid intersect. ${ }^{12}$ The findings that prosthetic eyes polished to an optical quality contact lens standard show more wettability than those polished to a normal standard, and that higher polished surfaces accumulate deposits at a slower rate, might be particularly important for the surface finish of the interpalpebral zone of the pros-

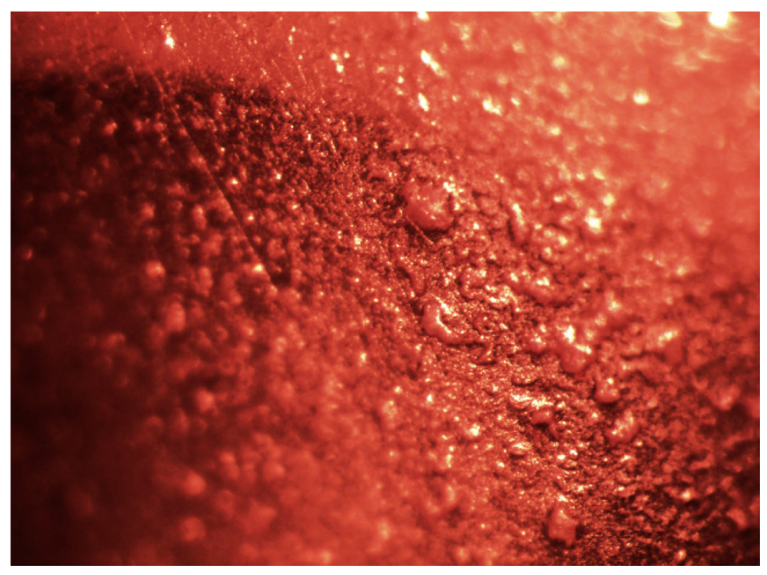

Figure 7 Deposits on the temporal limbus area of a left prosthetic eye worn continuously for 3 months (magnification $400 \times$ ).

Note: The dry deposits shown were stained with GC Corporation plaque disclosing gel. ${ }^{5}$ thesis. This is the zone where wetting and drying cycles occur and where the cleansing action of tears takes place. The interpalpebral zone needs to be as free from deposits as possible to avoid conjunctival battering during blinking. As the eyelids slide over the interpalpebral zone of a prosthetic eye containing deposits and debris, the area most likely to receive this battering is Marx's line ${ }^{13}$ which has been shown to develop epitheliopathy in contact lens patients with dry eye symptoms. ${ }^{14}$ Clearly, deposits left in the interpalpebral zone of prosthetic eyes are not likely to be beneficial to wearing comfort and, consequently, it is recommended that prostheses polished to an optical quality contact lens standard be the minimum standard for prosthetic eye finishes. An optical quality contact lens standard of finish may also facilitate the lubricating function of tears in the retropalpebral zone when the prosthesis is first inserted into the socket and before the layered coatings, films, or plaques of tear protein deposits become established. The finding that wetting angles decreased (and wettability increased) significantly when deposits were present in the retropalpebral zone may be the reason why surface deposits are associated with less severe conjunctival inflammation in anophthalmic sockets ${ }^{1}$ and that more frequent cleaning of prosthetic eyes (deposit removal) is associated with more severe discharge. ${ }^{15}$ By increasing surface wettability, the deposits may improve the ability of socket fluids to lubricate the prosthesis. If mucins are present in prosthetic eye deposits, as they are in contact lens deposits, ${ }^{16}$ components of glycoproteins such as the surfactant glycocalyx ${ }^{17}$ may also facilitate the lubricating function. The consequence of these properties of deposits 
would be that less frictional irritation of the conjunctiva occurs when deposits are present.

The finding that wetting angles of prosthetic eye surfaces decreased after only ten minutes of wear (suggesting the presence of protein deposits) is consistent with a number of observations reported in the contact lens literature. ${ }^{16,18-20}$

Limitations of the study include the short period of the 2-week experiment with different surface polishes (Figure 1) because this did not reveal how long the difference in deposit buildup rates for the two polishing standards might last. The investigation concerning the rate of deposit buildup on prosthetic eyes worn by two participants had limitations because of the small sample size. The reason for this small number was the invasive nature of the experiment coupled with the shortage of available participants who cleaned their prostheses less frequently than yearly.

\section{Conclusion}

Prosthetic eyes are somewhat analogous to contact lenses. Both devices come into contact with the conjunctiva, share a similar eyelid action, are bathed in the same ocular fluids, and accumulate surface deposits. Because of these similarities, relevant information from contact lens investigations has been included in this study. Two distinctive areas of deposit buildup are described. The first is the interpalpebral zone where deposits are exposed to the air and the action of the eyelids, and the second is the retropalpebral zone where deposits are in continuous contact with the conjunctiva. We recommend that an optical quality contact lens standard of finish (shown to have better wettability and a slower rate of deposit buildup) be the minimum standard of finish for prosthetic eyes. This standard may be important for the interpalpebral surface, where it assists the smooth action of the lids over the interpalpebral zone of the prosthesis and the cleansing action of tears. When deposits were removed by cleaning, surface wettability decreased significantly, suggesting that the presence of deposits in the retropalpebral zone improves the lubricating properties of socket fluids which, in turn, may result in less frictional irritation of the conjunctiva when the prosthesis moves. This finding provides evidence for a causal link between the presence of deposits and less conjunctival inflammation and discharge reported by Pine et al. ${ }^{2}$

\section{Disclosure}

Some of the participants in this study were recruited from the New Zealand Artificial Eye Service, which is owned and operated by Keith Pine. The authors report no other conflicts of interest in this work.

\section{References}

1. Pine KR, Sloan B, Jacobs RJ. The development of measuring tools for prosthetic eye research. Clin Exp Optom. June 6, 2012. [Epub ahead of print.]

2. Pine KR, Sloan B, Stewart J, Jacobs RJ. The response of the anophthalmic socket to prosthetic eye wear. Clin Exp Optom. 2012;6:707-713.

3. Franklin VJ, Tigue B, Tonge S. Contact lens care: Part 4 - Contact lens deposit build-up, discoloration and spoilation mechanisms. Optician. 2001;222:16-26.

4. Jones L, Evans K, Sariri R, Franklin V, Tighe B. Lipid and protein deposit build-up of $\mathrm{N}$-vinyl pyrrolidone-containing group II and group IV frequent replacement contact lenses. CLAO J. 1997;23:122-126.

5. Heiler DJ, Gambacorta-Hoffman S, Groemminger SF, Jonasse MS. The concentric distribution of protein on patient-worn hydrogel lenses. CLAO J. 1991;17:249-251.

6. Bego [homepage on the Internet]. Acrylic polish. Lincoln, RI: Bego USA; 2012. Available from: http://begousa.com. Accessed May 15, 2012.

7. Lin L. A concordance correlation coefficient to evaluate reproducibility. Biometrics. 1989;45:255-268.

8. Fowler SA, Korb DR, Finnemore VM, Allansmith MR. Surface deposits on worn hard contact lenses. Arch Ophthalmol. 1984;102:757-759.

9. Franklin VJ, Tighe BJ. Hydrogel lens spoliation. The structure and composition of white spot deposits. Optician. 1991;202:18-23.

10. LeGrand JA. Chronic exudate: an unnecessary evil. J Ophthalmic Prosthetics. 1999;4:33-40.

11. Weeks AK. Exploring the potential of hyaluronic acid as a wetting agent in model contact lens materials [dissertation abstract]. Hamilton, Ontario: McMaster University: 2011. Available from: http://digitalcommons. mcmaster.ca/dissertations/AAINR74084/. Accessed August 22, 2012.

12. Read ML, Morgan PB, Kelly JM, Maldonado-Codina C. Dynamic contact angle analysis of silicone hydrogel contact lenses. J Biomater Appl. 2011;26:85-99.

13. Donald C, Hamilton L, Doughty MJ. A quantitative assessment of the location and width of Marx's line along the marginal zone of the human eyelid. Optom Vis Sci. 2003;80:564-572.

14. Korb DR, Greiner JV, Herman JP, et al. Lid-wiper epitheliopathy and dryeye symptoms in contact lens wearers. CLAO J. 2002;28:211-216.

15. Pine KR, Sloan B, Stewart J, Jacobs RJ. A survey of prosthetic eye wearers to investigate causes of mucoid discharge. Clin Ophthalmol. 2012;6:707-713.

16. Fowler SA, Allansmith MR. Evolution of soft contact lens coatings. Arch Ophthalmol. 1980;98:95-99.

17. Liotet S, Triclot MP, Perderiset M, Warnet VN, Laroche L. The role of conjunctival mucus in contact lens fitting. CLAO J. 1985;11:149-154.

18. Franklin VJ. Cleaning efficacy of single-purpose surfactant cleaners and multi-purpose solutions. CLAO J. 1997;20:63-68.

19. Leahy CD, Mandell RB, Lin ST. Initial in vivo tear protein deposit build-up on individual hydrogel contact lenses. Optom Vis Sci. 1990;67:504-511.

20. Luensmann D, Jones L. Protein deposit build-up on contact lenses: the past, the present, and the future. Cont Lens Anterior Eye. 2012;35:53-64. 


\section{Publish your work in this journal}

Clinical Ophthalmology is an international, peer-reviewed journal covering all subspecialties within ophthalmology. Key topics include: Optometry; Visual science; Pharmacology and drug therapy in eye diseases; Basic Sciences; Primary and Secondary eye care; Patient Safety and Quality of Care Improvements. This journal is indexed on
PubMed Central and CAS, and is the official journal of The Society of Clinical Ophthalmology (SCO). The manuscript management system is completely online and includes a very quick and fair peer-review system, which is all easy to use. Visit http://www.dovepress.com/ testimonials.php to read real quotes from published authors. 